\title{
Relationship between viscosity and conductivity for tokamak plasmas
}

\author{
M. Asif \\ Department of Physics, COMSATS Institute of Information Technology, \\ Lahore 54000, Pakistan \\ (Received on 6 November, 2008)
}

\begin{abstract}
The relation between parallel viscosity and the parallel conductivity for tokamak plasma has been derived by combining Ideal MHD equilibrium equations with the concept of universal profiles. It is obtained that the parallel viscosity $\Pi_{\|}$and parallel conductivity $\sigma_{\|}$in a tokamak are related by $\Pi_{\|} \sigma_{\|}^{-1}=\gamma\left(U-U_{0}\right) I / R$, where $U$ is the loop voltage, $U_{0}$ is attributed to polarization, $I$ is the toroidal current and $R$ is the major radius of the machine. The coefficient $\gamma$ depends on para- or diamagnetism and on toroidal effects and exhibits a weak dependence on the minor radius.
\end{abstract}

Keywords: MHD equilibrium; viscosity; conductivity; current density

\section{INTRODUCTION}

As early as 1980, Coppi [1] pointed out that the electron temperature profiles in tokamaks tend to conserve their shape in response to an external action on the plasma. This effect, which was called "the profile consistency", was confirmed in many devices. Experimental observations and theoretical models supporting the idea that electron temperature and/or the pressure and/or density and/or current density in tokamaks preferentially assume certain privileged profile shapes have been reported for more than two decades $[1-8]$. The existence of universal current profiles in tokamaks $[2-3,9]$ and combining of Ideal MHD equilibrium equations with the concept of universal profiles has attracted interest among various authors. They have proposed the current density of a diffuse system $(0 \leq r \leq+\infty)$ by the formula

$$
j_{z}(r)=\frac{j_{z 0}}{\left(1+\mu r^{2}\right)^{2}}
$$

where $\mu=\pi j_{z 0} / I ; I=2 \pi \int_{0}^{\infty} r j_{z} \mathrm{~d} r$ is the toroidal current . This result was derived by minimizing the plasma energy under the condition that the toroidal current $I$ is kept fixed.

By combining the Ideal MHD equilibrium equations with the concept of universal profiles, it is shown by [8] that the electron density $n_{e}(r)$ and temperature $T_{e}(r)$ in a tokamak are related by $n_{e} T_{e}^{-1 / 2}=\alpha\left(U-U_{0}\right) I / R$ where $U$ is the loop voltage, $U_{0}$ is attributed to polarization, $I$ is the toroidal current and $R$ is the major radius of the machine. The coefficient $\alpha$ depends on para- or diamagnetism and on toroidal effects and exhibits a weak dependence on the minor radius. The above formula is in good agreement with ohmic and co-injection experiments performed in TEXTOR [10].

The aim of this paper is to develop a simple theory based relation between parallel viscosity and the parallel conductivity for tokamak plasma by combining Ideal MHD equilibrium equations with the concept of universal profiles. It is obtained that the parallel viscosity $\Pi_{\|}$and parallel conductivity $\sigma_{\|}$in a tokamak are related by $\Pi_{\|} \sigma_{\|}^{-1}=\gamma\left(U-U_{0}\right) I / R$, where $U$ is the loop voltage, $U_{0}$ is attributed to polarization, $I$ is the toroidal current and $R$ is the major radius of the machine. The coefficient $\gamma$ depends on para- or diamagnetism and on toroidal effects and exhibits a weak dependence on the minor radius.

\section{RELATION BETWEEN VISCOSITY AND CONDUCTIVITY}

Assuming cylindrical geometry with $B=\hat{e}_{\theta} B_{\theta}+\hat{e}_{z} B_{z}$ and $j=\hat{e}_{\theta} j_{\theta}+\hat{e}_{z} j_{z}$ we express Ampere's law and pressure balance as follows:

$$
\begin{gathered}
j_{\theta}=-\frac{c}{4 \pi} \frac{\partial B_{z}}{\partial r}, \quad j_{z}=\frac{c}{4 \pi} \frac{1}{r} \frac{\partial}{\partial r} r B_{\theta}, \\
\frac{\partial p}{\partial r}=\frac{1}{c}\left(j_{\theta} B_{z}-j_{z} B_{\theta}\right) .
\end{gathered}
$$

The current flowing in the $z$-direction through a section of radius $r$ is given by

$$
I_{z}(r)=2 \pi \int_{0}^{r} r_{1} j_{z}\left(r_{1}\right) \mathrm{d} r_{1}
$$

Substituting Eqs. (2) into Eq. (3) we obtain

$$
\frac{\partial}{\partial r}\left(p+\frac{B_{z}^{2}}{8 \pi}\right)+\frac{1}{4 \pi} \frac{B_{\theta}}{r} \frac{\partial}{\partial r} r B_{\theta}=0 .
$$

Multiplying Eq. (5) by $r^{2}$ and integrating by parts over the plasma section we get

$$
2 \pi \int_{0}^{a} r p(r) \mathrm{d} r=\frac{I_{z}^{2}(a)}{2 c^{2}}-\left(2 \pi \int_{0}^{a} r \frac{B_{z}^{2}(r)}{8 \pi} d r-\pi a^{2} \frac{B_{z}^{2}(a)}{8 \pi}\right) .
$$

However, we can write Eq. (6) as follows:

$$
2 \pi \int_{0}^{a} r\left(p(r)-\frac{I_{z}(a)}{2 c^{2}} j_{z}(r)+\frac{B_{z}^{2}(r)-B_{z}^{2}(a)}{8 \pi}\right) \mathrm{d} r=0
$$

and make the ansatz that the universal profiles nullify the integrand of Eq. (7) so that

$$
p(r)=\frac{I_{z}(a)}{2 c^{2}} j_{z}(r)-\frac{B_{z}^{2}(r)-B_{z}^{2}(a)}{8 \pi} .
$$


Eq. (8) is in fact an additional relation to the basic MHD equations (2) and (3) holding between the profiles. The radial derivative of Eq. (8) gives

$$
\frac{\partial}{\partial r}\left(p+\frac{B_{z}^{2}}{8 \pi}\right)=\frac{I_{z}(a)}{2 c^{2}} \frac{\mathrm{d} j_{z}}{\mathrm{~d} r} .
$$

On the other hand, by using Eqs. (4) and (2) the MHD equation (5) can be expressed as

$$
\frac{\partial}{\partial r}\left(p+\frac{B_{z}^{2}}{8 \pi}\right)=-\frac{2 j_{z} I_{z}(a)}{r c^{2}} .
$$

Combining Eqs. (9) and (10) we arrive at the following integrodifferential equation governing the current profile:

$$
r \frac{\mathrm{d} j_{z}}{\mathrm{~d} r}+\frac{4 I_{z}(r)}{I_{z}(a)} j_{z}=0, \quad I_{z}(r) \equiv 2 \pi \int_{0}^{r} r_{1} j_{z}\left(r_{1}\right) \mathrm{d} r_{1}
$$

It is easy to verify that the solution of Eq. (11) is

$$
j_{z}(r)=\frac{j_{z 0}}{\left\{1+\left[\pi j_{z 0} / I_{z}(a)\right] r^{2}\right\}^{2}},
$$

which has the same form as Eq. (1). Note, however, that solution (12) can satisfy only approximately the integral equation

$$
\int_{0}^{r} r j_{z}(r) \mathrm{d} r=I_{z}(a)
$$

because the system is now finite $(a \neq+\infty)$. This leads to the condition $\pi a^{2} j_{z 0} \gg I_{z}(a)$ which is necessary for Eq. (12) to be applicable in actual systems. The current profiles in this case must be rather peaked in the center.

Let us introduce $p=2 n_{e} T_{e}, j_{z}=\sigma_{\|} E_{z}$ and parallel viscosity is related to the pressure anisotropy, $\Pi_{\|}=\frac{2}{3}\left(p_{\|}-p_{\perp}\right)$ from Eq. (8) and obtain

$$
\Pi_{\|}\left(1+\frac{B_{z}^{2}(r)-B_{z}^{2}(a)}{12 \pi \Pi_{\|}}\right)=\frac{\sigma_{\|}}{3 c^{2}} I_{z}(a) E_{z},
$$

where $E_{z}=\left(U-U_{0}\right) / 2 \pi(R+r)$ is the induced electric field which drives the toroidal current $I=I_{z}(a)$. Here $U$ represents the loop voltage, $U_{0}$ could be attributed experimentally, and $R$ is the major radius of the tokamak. Absorbing further the quantity

$$
\left(1+\frac{B_{z}^{2}(r)-B_{z}^{2}(a)}{12 \pi \Pi_{\|}}\right)\left(1+\frac{r}{R}\right)
$$

in the coefficient $\gamma$ we finally get

$$
\Pi_{\|} \sigma_{\|}^{-1}=\gamma\left(U-U_{0}\right) I / R,
$$

where $\gamma$ depends on para- or diamagnetism and on toroidal effects.

Over the entire range of Ohmic discharges investigated on TCV tokamak [11], it is observed that the $\langle\sigma\rangle \approx\langle p\rangle$. These intriguing observations, although not central to the subjects developed in this article, is reminiscent of reports on electron temperature or pressure "profile consistency" $[12-13]$ reported from several experiments $[1,11-13]$. The relationship seen experimentally are consistent with Kadomtsev's [3] and Biskamp's [2] predictions based on the idea of plasma self-organization to a state of minimum energy. The relation $\langle\sigma\rangle \approx\langle j\rangle \approx\langle p\rangle$ has been shown to follow from the plasma equilibrium force balance [4]. It is important to note that the parallel viscosity which provides a finit contribution to plasma compressibility, is related to the pressure anisotropy.

\section{CONCLUSION}

By combining the Ideal MHD equilibrium equations with the concept of universal profiles, it is shown that the parallel viscosity $\Pi_{\|}$and parallel conductivity $\sigma_{\|}$in a tokamak are related by $\Pi_{\|} \sigma_{\|}^{-1}=\gamma\left(U-U_{0}\right) I / R$, where $U$ is the loop voltage, $U_{0}$ is attributed to polarization, $I$ is the toroidal current and $R$ is the major radius of the machine. The coefficient $\gamma$ depends on para- or diamagnetism and on toroidal effects and exhibits a weak dependence on the minor radius.This obtained relation may be of interest for practical applications because parallel viscosity provides a finit contribution to plasma compressibility.
[1] B. Coppi, Comm. Plasma Phys. Control. Fusion, 5 (1980) 261.

[2] D. Biskamp, Comm. Plasma Phys., 10 (1986) 165.

[3] B. B. Kadomtsev, Sov. J. Plasma Phys., 13 (1987) 443.

[4] E. Minardi, Phys. Lett. A, 240 (1998) 70.

[5] J. B. Taylor, Phys. Fluids B, 5 (1993) 4378.

[6] V. Arunasalam et al., Nucl. Fusion, 30 (1990) 2111.

[7] M. Asif, Int. J. Mod. Phys. B, 22 (2008) 5329.

[8] M. Psimopoulos, Phys. Lett. A, 162 (1992) 182.
[9] T. Y. Hsu and M. S. Chu, Phys. Fluids, 30 (1987) 1221.

[10] H. Soltwisch, W. Stodiek, T. Manickam and T. Schluter in : Proc.11th Conf. on plasma physics and controlled nuclear fusion research , Kyoto, Japan (1992).

[11] E. Minardi, Nucl. Fusion, 37 (1997) 1741.

[12] H. Weissen, Phys. Plasmas, 6 (1999) 1.

[13] Y. N. Dnestrovskij, Plasma Phys. Rep., 28 (2002) 963. 